\title{
Influence of solid matrices on spectral features of metalloporphyrins
}

\author{
Aleksandr Starukhin ${ }^{1, *}$, Aleksander Gorski ${ }^{2}$, and Michal $\mathrm{Kijak}^{2}$ \\ ${ }^{1}$ B.I. Stepanov Institute of Physics, National Academy of Sciences of Belarus, Minsk 220072, Belarus \\ ${ }^{2}$ Institute of Physical Chemistry, Polish Academy of Sciences, Warsaw 01-224, Poland
}

\begin{abstract}
A way of simultaneously forming in the ground electronic state a planar, as well as two distorted forms was established for a set of metalloporphyrins in solid matrices at cryogenic temperatures. Experimental data together with results of theoretical DFT calculations are combined for analysis and assignment of possible types of distorted porphyrin macrocycles conformations.
\end{abstract}

Metallocompexes of porphyrins are biologically relevant compounds known for many years. On other side metalloporphyrins are suitable compounds for single molecule spectroscopy as molecules with wide variable different spectral features at cryogenics temperatures. However, despite intensive studies by many groups, their spectral features and photophysical properties are still the subject of long debates and new experiments. The spectroscopic studies at low temperatures belong to effective and proven methods of investigation of fine details such as a vibrational structure of molecules in both the ground and excited states. One of the most spectacular methods for recording fine-line luminescence spectra is the fluorescence line narrowing (FLN) [1].

In this report, we discuss the results, obtained for set of the simplest metalloporphyrins in different solid solutions, crystals and rare gas matrices at liquid helium temperature. The interest in such type of study was motivated by a unique possibility to obtain the spectra of the different spectral conformation of metalloporphyrins.

For example, fluorescence spectra of three different spectral forms Zn-porphine (ZnP), embedded in solid argon matrix are presented on Fig. 1. Fluorescence excitation spectra were recorded upon detection of fluorescence bands for corresponding spectral conformation also. These spectral features are caused by realization the structures with position of Zn- atom «in plane»» (Form F0 - Fig1a) and two types «out of plane» of porphyrin macrocycles in matrices (Form FI- Fig1b and FII- Fig1c) [2]. Above mentioned forms of $\mathrm{ZnP}$ in argon matrix have the different positions of the 0-0 transitions of the first electronic states at 552.3, 562.4 and $566.45 \mathrm{~nm}$. The energy gap between positions of the 0 0 transitions more than $300 \mathrm{~cm}^{-1}$ of Form F0, for Form FI. Spectral gap beween Form F0 and Form FII have the value about $450 \mathrm{~cm}^{-1}$. In a Soret band range the position of longwave transitions for three forms are manifested at 385, 388 and $404 \mathrm{~nm}$, correspondingly.

\footnotetext{
* Corresponding author: starukhin@mail.ru
} 

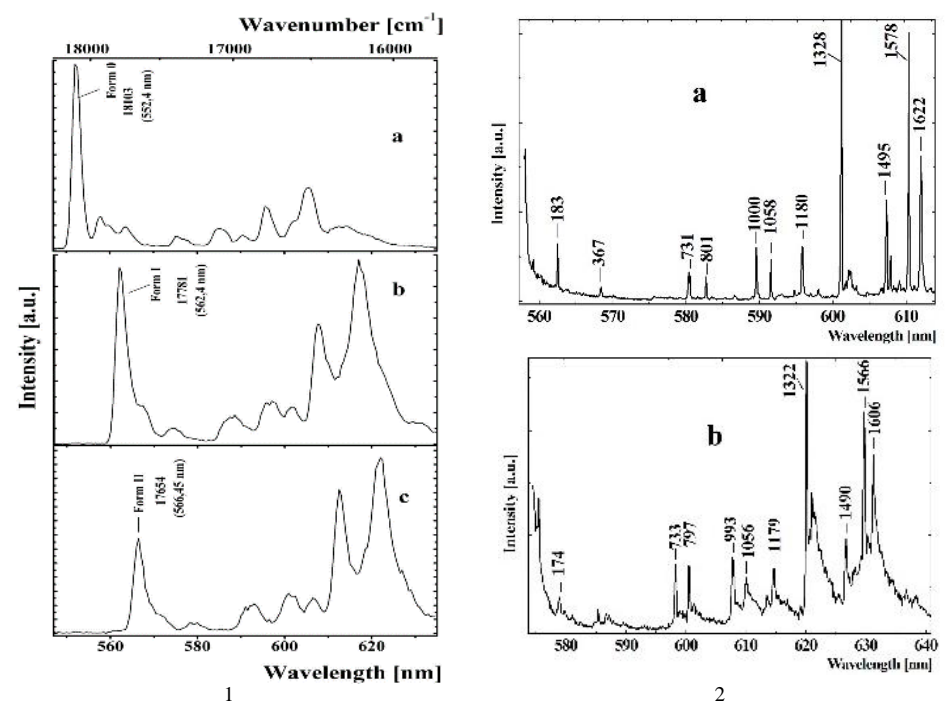

Fig. 1. Fluorescence spectra of $\mathrm{ZnP}$ (1) in solid argon matrix onto a sapphire substrate under nonselective excitation: $\lambda_{\text {exc. }}=385(\mathrm{a}), 388(\mathrm{~b}), 404 \mathrm{~nm}$ (c) at $\mathrm{T}=5 \mathrm{~K}$ and FLN spectra (2) of ZnP in $n$-octane, $\lambda_{\text {exc. }}=556,8 \mathrm{~nm}$ (a) and $\mathrm{ZnP}$ in $n$-octane with pyridine $\lambda_{\text {exc. }}=573,1 \mathrm{~nm}$ (b) at $4,2 \mathrm{~K}$

Relative intensities of the $0-0$ and vibronic transitions are very different for these forms (Fig. 1). Upon the fluorescence line narrowing conditions there are essential difference in intensities of vibronic transitions. It is connected with different character of vibronic borrowing intensities for vibrational transition upon formation of vibrational structure of fluorescence spectra for above-mentioned forms. FLN spectra of Form F0 (realized for ZnP in $n$-octane) and Form FII ( $\mathrm{ZnP}$ in $n$-octane with pyridine) were recorded upon laser selective excitation. The modes with frequencies 1578 and $1622 \mathrm{~cm}^{-1}$ (Form F0) decrease to 1566 and $1606 \mathrm{~cm}^{-1}$ in spectrum of Form FII, correspondingly. An empirical interpretation of this effect can lead to wrong conclusions Density Functional Theory (DFT) and time dependent DFT methods were used for optimization of the ground and excited states together with calculation intensities and frequencies of normal modes of mettalocomplexes. Based on performed calculations, an assigmentof experimentally observed forms was proposed. The FI form was attributed to a complex of metalloporphyrin with one extra ligand placed above its plane. The FII form corresponds to a structure with two additional ligands located at opposite sides of the porphyrin macrocycle. Complexes of metalloporphyrins with rare gas may be realized as Van der Waals complexes between metalloporphyrin and rare gas. Upon condensation of the molecules into rare gas matrix, the distance between the matrix and the chromophore is less than one nanometer, which can lead to a significant increase of the probability of Van der Waals complexes formation [3].

This work was supported by the European Union's Horizon 2020 research and innovation program under grant agreement № 645628 and project № F18RA-012.

\section{References}

1. R. I. Personov, E. I. Al'Shits, L. A. Bykovskaya, Opt. Commun. 6, 169 (1972)

2. A. S. Starukhin, A. M. Shul'ga, Opt. Spectrosc. 98, 780 (2005)

3. U. Barash, V. L. Ginzburg, Physics-Uspekhi 143, 345 (1984) 